\title{
Identification of the Area for Proper Integration of Three Current Storage Objects into One Complex Logistics Point
}

\author{
Chenguang $\mathrm{Li}^{1}$ and Martina Hlatká ${ }^{2 *}$
}

${ }^{1}$ School of Economics and Management, North China University of Technology, No. 5 Jinyuanzhuang Road, Shijingshan District, Beijing 100144, The People 's Republic of China; Email:dr.lcg@qq.com

${ }^{2}$ Department of Transport and Logistics, Faculty of Technology, Institute of Technology and Business in Ceske Budejovice, Okruzni 517/10, 37001 Ceske Budejovice, Czech Republic; Email: hlatka@mail.vstecb.cz.

\section{*Corresponding author: Martina Hlatká}

\begin{abstract}
The paper presents an option to identify the suitable area for locating the specific storage object in order to integrate three current storage objects into one complex logistics point. Initial chapters of the paper are focused on overview of theoretical terms related to the storage objects, their activities, services, parameters as well as their location and allocation. Other parts of this paper outline specific methods regarding the issue of storage objects location. Its main part describes individual steps for identifying the suitable storage object location in the certain area using the specific method.
\end{abstract}

Keywords: Storage object, integration, logistics, identification, location

\section{Introduction}

Storage logistics is the link between the production and sales of a business. It includes all stock movements and transport of the goods to the purchaser (customer) and related information, management and control activities. The aim is to make available the right goods at the right time at the right place in the right quantity and quality and at the same time to create an optimal ratio between a given set of delivery services a company is able to provide, or is required to provide by the customer, and the emerging costs. Storage logistics means the correct serving of warehousing channels. For enterprises, an increasing trend is to use the allocation of their products, in addition to other tools of distribution policy, as a competitive tool, which enables them to gain a competitive advantage with regards to improved service delivery [1,2].

It is also necessary to take into account the requirements of customers which mostly include the provision of additional services such as storage and the preparation of their products. Customers increasingly look to reduce their own stocks, and for these reasons, prefer to order in smaller 
quantities and at shorter intervals with maximum synchronization. This makes the supply companies develop appropriate delivery strategies that ensure high delivery readiness and alertness, without increasing costs at the same time.

Using mathematical operations research methods, it is possible to locate servicing points in order to minimize the material costs and cost of current organizational links, production units, buildings and equipment. To solve the nature of this problem, several approximation methods, often very complex ones, have been proposed [3-11].

\section{Theoretical Definition of Storage Objects}

The main purpose of a storage object is the optimal interconnection of means of transport in order to generate optimal traffic flows and network storage objects for each region. Storage objects integrate several logistics providers with their intense logistical requirements, leasing, insurance and banking companies in one place $[7,8]$.

For the implementation of the transport requirements a storage object uses multiple modes of transport (especially road/rail but also inland waterway transport) with regards to the systemic benefits of each means of transport. Furthermore, it manages and enhances cooperative relationships between different entities. Spatial networking of carriers and shippers creates synergies, especially in sharing infrastructure for combined transport in the development of intermodal transport chains at the national and international level. The aim is to ensure sustainable development in the ever growing traffic and to constantly seek ways to relieve the pressure on the road infrastructure [7-9].

\subsection{Benefits of Storage Objects}

A wide range of business sectors in a storage object, and a logistically oriented range of services, creates favorable conditions for the conclusion of new trade agreements. The management of storage objects supports the development of innovative products in businesses located in the storage objects $[7,10]$.

By effective management of logistics activities, a storage object reduces the pressure on the transport infrastructure and makes transport more environmentally friendly, whilst at the same time stimulating changes in infrastructure in line with the forecasted requirements of the logistics market. The benefits cannot be evaluated in isolation at the regional level; it increases through links to other storage objects which cooperate with each other at various levels [1]. 


\subsection{Definition of Basic Conditions}

Ten basic conditions necessary for the establishment of a storage object ( $\mathrm{SO}$ ) are identified as follows [7,8]: 1) geographic location determines the $\mathrm{SO}$ as a link between commodity flows, 2) in terms of competition the SO must be the best, 3) trimodal transport links are a great advantage for a SO,4) trimodal transport network ensures adequate commodity flows, 5) enough space for the planned construction of the SO is a prerequisite for a reasonable price, 6) construction of an expertly conceived SO requires a development department (a separate entity) which acts as a professional representative for all the business entities located in the SO and must be in close contact with the main construction planning object, 7) enhanced competitive edge of the SO is gained even before opening if it is well connected to the public transport infrastructure, 8) Silicon Valley effect is accelerated by a concentration of logistics companies with a large number of employees, 9) degree of vertical and horizontal interdependence provides potential for synergies, 10) sufficient storage space for logistics, light manufacturing and wholesale.

The construction of storage objects is affected by the growth of the range of transport modes, the growing importance of combined transport and the related restructuring of the logistics of loading, reloading and shipment $[9,10]$.

\section{Problem of Location and Allocation}

Location: the problem of where to place storage objects within the transport network. Allocation: the problem of assigning objects to be served by the individual objects (creating attraction circuits).

Location-allocation problems: the decision on the allocation of service centers.

ALA method: a method based on changing location and allocation; the task is to solve the problem of optimum allocation of the objects to be served by objects, while the location of these objcts is known; the second goal is to solve the problem of the optimal location of service centers when the this objects of attraction circuits are given [2,3,11].

\subsection{Methods for Deploying the Storage Object Solutions}

Among the tools utilized to determine the deployment of a SO, allocation, geometric and multicriteria methods are known [11].

The allocation method deals with the deployment of objects, location of a manufacturing process or a business, location of a SO, distribution of manufacturing operations at the workplace or placing machinery and equipment. The limiting conditions are the transport costs, time of distribution and other related activities. The solution is the location of a new object within the existing network or the selection of one or more objects from an existing network $[7,8]$. 
The geometric method works on the principle of the "Center of Gravity". The method helps to determine the geometric coordinates of where the specific SO should be located. The objective function in this methodology is minimal, it deals with costs minimization. The input data includes coordinates, size, material flow among objects and the future object, the material flow costs and the distance of the objects from the central point. Distance can be axial, square, straight and corrected. The direct distance is used in aviation transport, corrected distance is used in road and railway transport. If more than one object is to be located at the same time, the ALA method is utilized $[5,6]$.

Multi-criteria methods are used when placing a SO in existing hubs. The multi-criteria evaluation of variants uses multi-criteria programming. The objective function is minimal; it deals especially with the minimization of transport costs, minimization of the cost of vehicle travel time, and the minimization of $\mathrm{CO}_{2}$ emissions for all vehicles [7,11].

\subsection{Location of Storage Object}

According to the shape of the objective function, we distinguish the location of the median and the SO. The purpose of the location of the median is to find such a location that minimizes the sum of the weighted distances of all objects served from the nearest object. The objects are assessed by weight. This means that the graph $G=(V, H, C, w)$ tries to find the peak $m$ for which the minimum function is (Eq. 1) [5-7]:

$$
f(m)=\sum w(v) d(m, v)
$$

where: $C(\mathrm{~h})$ is the evaluation of edges [-],w (h) is the weight of the peak [-] and $d(m, v)$ is a distance of $m$ from $v[\mathrm{~km}]$.

If the subject of the method solution is the location of the median, the purpose is to find a location that minimizes the maximum distance of each facility operated from the nearest object. In the graph $G=(V, H, \mathrm{c})$, the objective is to find the top $m$ for which the minimum function is $(E q .2)$ $[6,7]$ :

$$
f(m)=\max \{d(m, v): v \subset V\}
$$

\subsection{Fermat-Weber Location Problem}

When solving this problem, the input data is represented by geometric coordinates $m$ of the served objects $\left[a_{i}, b_{i}\right]$, weight evaluation of objects $W_{i}$ and Euclidean space. The objective is to find the location of the median in the network of coordinates, i.e. to find the coordinates $[x, y]$ which minimize the function (Eq. 3) [6-8]: 


$$
f(x, y)=\sum_{i=1}^{m} W i \sqrt{\left(\begin{array}{ll}
x & a_{i}
\end{array}\right)^{2}+\left(\begin{array}{ll}
y & b_{i}
\end{array}\right)^{2}}
$$

\subsection{Warehouse Location Problem}

The warehouse location problem is used for discrete locations. The input elements are the number of customers $m$, the demands of customers $r_{i}$, the potential locations of warehouses $p$, the fixed costs of setting up and operation of warehouses $f_{i}$, unit costs $v_{i j}$ of transport from the warehouse $j$ to the customer $i$. The method solves the problem of deciding which warehouses will be established and the allocation of individual customers to them. The mathematical model describes the minimization function (Eq. 4) [5-7,9,10]:

$$
z=\sum_{j=1}^{p}\left(f_{i} y_{j}+\sum_{i=1}^{m} v_{i j} r_{i} x_{i j}\right)
$$

Under the conditions: $\sum_{i=1}^{p} x_{i j}=1$, where $i=1, \ldots, \mathrm{m}, y_{j} \geq x_{i j}$, where $i=1, \ldots, \mathrm{m} ; j=1, \ldots, \mathrm{p}$, $\mathrm{O} \leq x_{i j} \leq 1$, where $i=1, \ldots, \mathrm{m} ; j=1, \ldots, \mathrm{p}, y_{j} \in\{0,1\}$, where: 1 means that the warehouse will be established and 0 means that the warehouse will not be established.

\subsection{Weiszfeld Algorithm}

The Weiszfeld algorithm is designed to solve locational problems in Euclidean space. During the calculation, it converges to the optimum. The principle of coordinates transformation $W(x, y)$ converts the coordinates of the point $[x, y]$ into the coordinates $\left[x_{1}, y_{1}\right]=\mathrm{W}\left[x_{0}, y_{0}\right],\left[x_{2}, y_{2}\right]=W\left[x_{1}\right.$, $y_{1}$ ]. It is desirable to end the calculation before reaching the limit. The starting coordinates can be selected arbitrarily and do not affect the quality of the solution.

The calculation of distance between two points is calculated according to the equation $(E q .5$ and 6) $[4,7,9]$ :

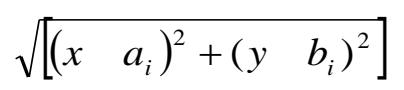

The total costs of the objects operation are calculated according to the equation:

$$
f(x, y)=\sum_{i=1}^{m} W i \sqrt{\left(\begin{array}{ll}
x & a_{i}
\end{array}\right)^{2}+\left(\begin{array}{ll}
y & b_{i}
\end{array}\right)^{2}}
$$

Partial derivatives $f(x, y)$ are calculated by $x$ and $y$, where the equation set is equal to 0 and 
expresses the variables $x$ and $y$.

\section{Particular Method Selection}

For the given research study, "Center of Gravity" was selected which is one of the methods used to identify the storage objects location and is applied mainly within projecting the warehouse locations situated between production and consumption. In line with this strategy, SO are placed approximately in the middle between the producer and the consumer.

In this case, the level of customer service is usually higher compared to the SO location strategy based on production and lower than with the strategy of warehouse location based on the market. This strategy is appropriate to ensure the higher customer service level when the range of products manufactured at different production sites is broad $[5,6]$.

\subsection{Center of Gravity Method}

This method is also called "The method of coordinates". It is suitable to determine the optimal spatial location of a central storage object which cooperates with several already placed objects [57].

The principle of the method is to find the optimal location of a SO in the center of gravity of a rectangular coordinate system constituted by other already existing objects or operations. In the Euclidean plane, $n$ points are indicated and labeled $A_{1}, A_{2}, \ldots, A_{n}$. At this plane, it is necessary to find a point $T$ for which the sum of given distances (given by $q_{1}, q_{2}, \ldots, q_{n}$ ) of the points $A_{1}, A_{2}, \ldots$, $A_{n}$ from point $T$ is the least possible [5,6,11].

The $X$ and $Y$ coordinates identifying the center of gravity $T$, i.e. the potential location of the central storage object in the selected coordinate system, is calculated as follows (Eq. 7 and 8) [5,6]:

$$
X=\frac{\sum x_{i} q_{i}}{\sum q_{i}}
$$

and

$$
Y=\frac{\sum y_{i} q_{i}}{\sum q_{i}}
$$

where: $x_{i}, y_{i}$-coordinates of the $\mathrm{i}$-th situated object, $q_{i}$ - transported volume per unit of time of the $i$-th situated object. Coordinates calculated by this method will specify the optimal location (area) of the main storage object on the basis of the input data. 
4.2 Identifying the Proper Area of the Central Storage Object in Central and Eastern Europe.

A survey was performed on the basis of requirements of ČSAD Hodonín, a.s enterprise which provided input data. The objective of this research study was to specify the appropriate location of a central storage object which should integrate three existing storages into one point in order to operate the Czech Republic, Slovakia, Poland and Hungary, and also serve as the main storage object for other countries in Central and Eastern Europe.

Input data:

- Flow rate per unit time in the $i$-th specified regional SO (gross weight) by postcodes;

- Manufacturing plants with the amount of goods needed to satisfy the market in Central Europe;

- Map of Europe with the division of territorial units according to the national postcodes to accurately determine the coordinates according to the regional SO postcodes (see Calculations Table 1):

Table 1 Gross weight deliveries to the $i$-th regional storage objects by postcodes for the year 2009, offsetting factory supply in Central Europe $\left(q_{i}\right)$. Source: [authors]

\begin{tabular}{c|c|c|c|c|c|c}
\hline City & Area postcode & $\sum q_{i}$ & $x_{i}$ & $y_{i}$ & $x_{i} \sum q_{i}$ & $y_{i} \sum q_{i}$ \\
\hline Ostrava & $70-74$ & 207152 & 11.8 & 12.3 & 2444393.6 & 2547969.6 \\
\hline Warszawa & 0 & 1998220 & 16.1 & 19.8 & 32171342.0 & 39564756.0 \\
\hline Bratislava & 8.90 & 224088 & 9.8 & 7.1 & 2196062.4 & 1591024.8 \\
\hline Budapest & 1.2 & 2796270 & 13.7 & 5.7 & 38308899.0 & 15938739.0 \\
\hline
\end{tabular}

The coordinates $x_{i}$ and $y_{i}$ are the point $A_{i}$ coordinates (storage objects in particular cities) in an artificial coordinate system (see Fig. 1). Subsequently, the calculation of $T$, and its coordinates $X$ and $Y$, allows us to specify the optimal spatial location of the central storage object cooperating with several already placed objects.

The total input values for calculating coordinates are:

$$
\sum q_{i}=474963447
$$

The product of values $x_{i} \sum q_{i}$ is:

$$
x_{i} \sum q_{i}=460053356.8
$$

And $y i^{*} \sum q i$ for the coordinate $Y$ is:

$$
y_{i}^{*} \sum q_{i}=568637677.3
$$

Table 1 contains only the selected values from the complex calculation. The calculation of the 
coordinates of the point $T$ according to equations (7) and (8) is as follows:

$$
X=9.68 \quad Y=11.97
$$

The calculated coordinates of $T$ have been transferred to Fig. 1 for illustration purposes. It can be concluded that, using the Center of Gravity method, based on the input data and the calculation of the central storage objects, location Central Moravia should be selected as the suitable area [8,9].

The proximity and good transport connections to a plant in Vizovice is another equally important factor which can confirm that the central SO is located correctly. The manufacturing plant in Vizovice produces material intended for consumption in the Eastern European market. This research study proves that the location specified by our calculations for the SO is the correct selection in regard to the future development.

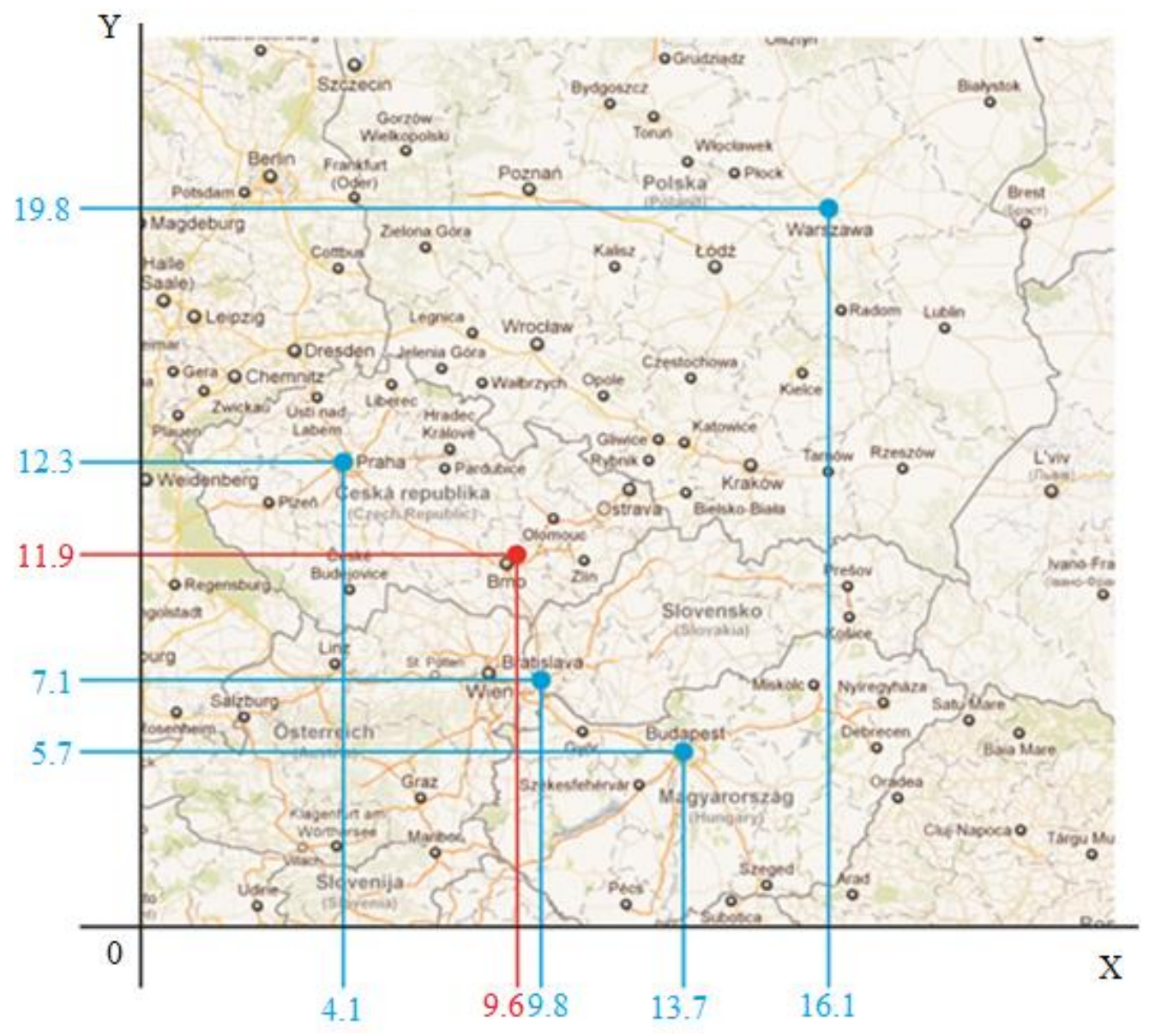

Fig. 1 Selected locations coordinates. Source: [authors, based on www.google.com/maps/]

\section{Conclusion}

The Center of gravity method was used to identify the suitable area for a central storage object that will integrate three existing storages into one complex logistics point in order to operate the Czech Republic, Slovakia, Poland and Hungary, and also serve as the main storage object for other 
countries in Central and Eastern Europe.

The Center of gravity method was selected both in regard to the objective, i.e. specifying the suitable position of the central storage object as well as in regard to the provided data.

When identifying the location of the storage object, storage of goods under normal and special conditions (hazardous material), guarantee of quality of logistics services, IT equipment and last but not least the experience and training levels of staff were taken into consideration.

According to the calculations, Brno is the most relevant area for location of the central storage object, due to its excellent location in central Europe at the crossroads of major road and railway transport networks.

Brno is a dynamic metropolis in South Moravia and has a long industrial tradition and high quality industrial base which offers a wide range of possibilities for cooperation. Its diversity and environmental friendliness represent the big advantage of the industry.

Brno is situated at the crossroads of the D1 (Prague - Brno - Ostrava - Poland) and D2 (Brno Slovakia). Both motorways are part of the east - west trans-European highway (France - Ukraine: E50) and north - south trans-European highway (Scandinavia - Balkans: E55 and E65).

Brno belongs among one of the most important railway stations in central Europe, situated in the international railway route Balkans - Budapest - Brno - Prague - Berlin - Scandinavia.

Railway lines from Brno are heading in these directions:

- Brno - Česká Třebová - Pardubice - Prague

- Brno - Tišnov - Havlíčkův Brod - Prague

- Brno - Znojmo, Jihlava

- Brno - Přerov

- Brno - Veselí nad Moravou - Trenčianská Teplá

Based on the above mentioned, Brno is considered the suitable area in order to locate the central storage area.

\section{References}

[1] Almetova, Z., Shepelev, V. \& Shepelev, S. (2016). Cargo transit terminal locations according to the existing transport network configuration. Procedia Engineering, 150(2016), 1396-1402. DOI: $10.1016 /$ j.proeng.2016.07.335.

[2] Liu, X., Zhao, X., Xue, Y. \& Zhu, C. (2012). Optimized location planning of logistics park based on implicit enumeration. Advances in Intelligent and Soft Computing, 135, 327-332. DOI: 10.1007/978-3-642-27708-5_43. 
[3] Hong, X. (2011). Methods to determine logistics distribution center location. International Conference on Management and Service Science, Accession Number: 12216660, Wuhan, China. DOI: 10.1109/ICMSS.2011.5998301.

[4] Velasquez-Marti, B. \& Fernandez-Gonzalez E. (2010). Mathematical algorithms to locate factories to transform biomass in bioenergy focused on logistic network construction. Renewable Energy, 35(9), 2136-2142. DOI: 10.1016/j.renene.2010.02.011.

[5] Liu, S.J., Wang, Z., Miao, R., Xu, J. \& Huang, H. (2013). Research of location selection of distribution center for service based on gravity method. Applied Mechanics and Materials, 433-435, 2419-2423. DOI: 10.4028/www.scientific.net/AMM.433-435.2419.

[6] Chen, Z.X. \& He, W. (2010). Study and application of Center-of-Gravity on the location selection of distribution center. International Conference on Logistics Systems and Intelligent Management (ICLSIM), 2, Article number 5461101, 981-984. DOI: 10.1109/ICLSIM.2010.5461101.

[7] Gopalakrishnan, M., Narayanan, S., Bodner, D.A., Patchigolla, K.Y., Kantamneni, R.P., Edala, N.R. \& Ruff, H.A. (2001). A computerized system for storage location assignment in third party warehouses. International Journal of Industrial Engineering: Theory, Applications and Practice, 8(2), 159-167. ISSN 1072-4761.

[8] Ochmanska, E. (1997). Object-oriented PN models with storage for transport and logistic processes. 9th European Simulation Symposium (ESS 97), Passau, Germany, 483-487.

[9] Fontana, M.E. \& Cavalcante, C.A.V. (2014). Use of Promethee method to determine the best alternative for warehouse storage location assignment. Internation Journal of Advanced Manufacturing Technology, 70(9-12), 1615-1624. DOI: 10.1007/s00170-013-5405-z.

[10] Yang, C.L. \& Nguyen, T.P.Q. (2016). Constrained clustering method for class-based storage location assignment in warehouse. Industrial Management \& Data Systems, 116(4), 667-689. DOI: 10.1108/IMDS-09-2015-036.

[11] da Silva, D.D., de Vasconcelos, N.V.C. \& Cavalcante, C.A.V. (2015). Multicriteria Decision Model to Support the Assignment of Storage Location of Products in a Warehouse. Mathematical Problems in Engineering, Article Number: 481950. DOI: $10.1155 / 2015 / 481950$. 